\title{
Monarch Bt-corn paper questioned
}

The May publication in Nature of a peerreviewed Scientific Correspondence paper indicating that transgenic pollen kills larvae of the monarch butterfly provoked contrasting responses around the world. While environmental interest groups seized on the findings as demonstrating the harmful effects of genetically modified crops on "nontarget" species in agricultural regions, plant scientists and representatives of life science companies among others criticized the work as premature, incomplete, and unconvincing.

In the controversial experiments, monarch larvae fed on milkweed leaves dusted with pollen from N4640-Bt corn (a commercial variety containing a gene for an insecticidal Bacillus thuringiensis protein as a defense against infestation with the European corn borer) ate less, grew more slowly and had higher mortality than those fed plain leaves or leaves dusted with pollen from a nontransgenic corn line. After four days, survival of larvae exposed to N4640-Bt corn was $56 \%$ compared with $100 \%$ for the pollen-plus and pollen-minus controls. The monarch, whose larvae feed exclusively on milkweed leaves, is regarded as a particularly sensitive indicator of environmental disturbance.

Critics of the work have particularly highlighted a number of weaknesses of the paper. The first is that it is unsurprising that a lepidopteran species such as the monarch should be affected when fed plant material that contains a protein used precisely because of its lepidopteran-specific killing properties.

Willy De Greef, the worldwide head of regulatory and government affairs for Novartis Seeds (Basel, Switzerland), the company that developed the N4640-Bt variety, would have liked to see a greater stringency with respect to the Cornell University (Ithaca, New York) group's experiments. The forced feeding of a potentially toxic compound to larvae does not really represent the reality in the field. More convincing, he says, would have been the results of "choice" experiments. where, as in the field, the monarch larvae are exposed simultaneously to milkweed leaves with and without $B t$-maize pollen. The preliminary Cornell experiments reported in Nature indicate that the presence of both transformed and untransformed pollen reduces leaf consumption by the larvae, something that might be expected to have a bearing on larval survival. De Greef says that the "choice" experiments would explore the monarch's pollen avoidance strategies.

The second major criticism is that the experiments were poorly quantified. At the
European Plant Biotechnology Network's Phytosfere meeting held in Rome at the beginning of June, a show of hands in a plenary discussion indicated that the majority of delegates resoundingly rejected the work's validity. "If I had measured out pollen by dropping it onto leaves with a spatula [the method the Cornell researchers used]", said one delegate from the Netherlands, "I would expect to be chopped into little pieces during peer review." John Losey, the lead author on the paper, admits that the methods used were "not stringently quantitative", but he says that there is "no reason to believe that there would be a systematic error in one direction or the other."

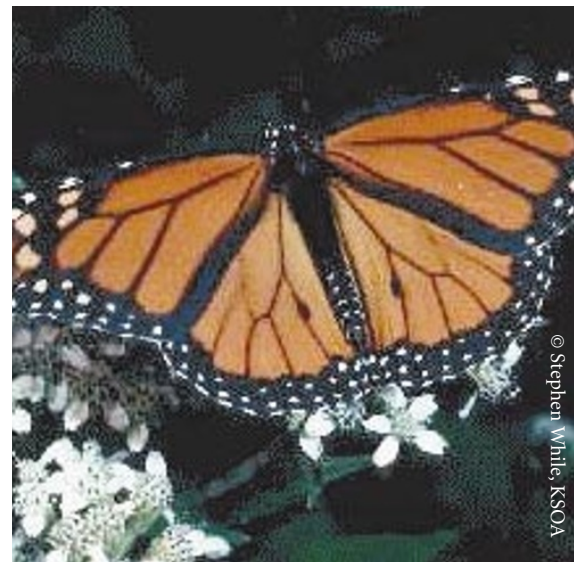

Plant scientists and representatives of life science companies criticized the work as premature and incomplete.

De Greef of Novartis also considered that the data presented could not be used to come to any conclusion about the real effect of $\mathrm{Bt}$ toxin-containing pollen even on monarch larvae. "The impact would depend how much of the milkweed available to the monarch larvae was affected," he said. "If it was $75 \%$, then this might be important: if it was $10 \%$, then it would probably not be important." Losey says that this element will be corrected by the work that the Cornell group will do this summer and next. "The dose [of pollen] we used was set to be high-the milkweed was taken right from within the cornfield." he says. "In the next phase, we want to quantify the concentration of pollen at various distances from the field. Then we can do a dose-response for each hybrid [corn]."

Another possible gap in the Cornell work is the use of inappropriate controls. As their pollen-plus control, the Cornell group used an unrelated corn hybrid rather than the isogenic variety on which N4640-
$B t$ was based. "We used it because it was there ..." says Losey. "[It was] available to us when we were collecting materials." However, the Cornell group is planning experiments this summer that will include the N4640-Bt isogenic line. Losey does not expect that this will influence the outcome of the experiment: "I will be very surprised if there is any significant difference."

Losey also admits that the preliminary work gathered no direct evidence that the $B$. thuringiensis toxin in the maize pollen was the cause of death. "There is no particular [external] pathology symptomatic of death by Bt." He says. "All we know is that the larvae died and were smaller than the controls." Some of the Cornell group's unpublished data throw up another interesting thread that needs to be untangled in subsequent work. Apparently, caterpillars feeding on leaves dusted with nontransgenic pollen grew significantly larger than those on the leaves with no pollen. "It's as if the pollen provides a little extra package of nutrients," explained Losey. In fact, it seems likely that that this "extra package" formed a substantial component of the larval diet. The pollen-plus control larvae got fatter even though they consumed only $70 \%$ as much leaf area as the pollen-minus control larvae (data published in Nature 399, 214).

If there are any effects from Bt-corn on nontarget organisms - and the Cornell study does not establish that convincingly at this point - any risk assessment should proceed on a relative basis. That assessment will not be simple. According to Losey, in those fields that are going to be treated against the European corn borer, Bt-corn clearly is the most environmentally sound option. However, that still may not make it the preferred option. The European corn borer is very hard to treat conventionally with insecticides. It has to be treated either early-before its second instar when it bores into the corn stalk-or late in the corn-growing season when second generation insects emerge. Late spraying requires specialized equipment or airplanes, and although late spraying is effective, it can only realistically be performed on large fields away from human habitations or roads. The net result is that less than $20 \%$ of the corn crop is actually treated. Thus, adverse environmental effects from conventional treatments only accrue to $20 \%$ of corn plantings. In contrast, $B t$ corn already represents $30 \%$ of the area, and this proportion is increasing.

John Hodgson 